\title{
Avaliação in vitro do potencial cariogênico e erosivo da Dipirona
}

\author{
In vitro evaluation of the caryogenic and erosive potential of dipyrone \\ Evaluación in vitro del potencial cariogénico y erosivo de dipirona
}

Resumo

O sucesso dos tratamentos odontológicos, especialmente na odontopediatria, está diretamente ligado ao plano de tratamento, onde devemos considerar o paciente como um todo, inserido dentro de um contexto familiar e influenciado por sua realidade social. Dentro destes aspectos, tratamentos minimamente invasivos e principalmente o controle da dor são fundamentais para o estabelecimento de uma relação harmoniosa entre paciente, profissional e família. O Cirurgião dentista pode utilizar uma extensa lista de medicamentos que podem atuar no auxílio do controle da dor, dentre os analgésicos mais utilizados, destacamos a Dipirona. Para este estudo foram utilizadas 04 amostras do analgésico Dipirona que foram divididos de acordo com os fabricantes: G1 - Medley (genérico), G2 - Neo Química, G3 - EMS e G4 - Medley (Novalgina). Após a manipulação dos medicamentos de acordo com o fabricante foram aferidas amostras de $\mathrm{pH}$ de cada laboratório em diferentes tempos (imediatamente, 48 horas e 7 dias). Após análise de Variância e Teste de Tukey os resultados demonstraram que não foram encontradas diferenças estatisticamente significativas no $\mathrm{pH}$ dos diferentes tempos de um mesmo laboratório, porém ao se considerar os diferentes laboratórios, todos apresentaram diferenças estatisticamente significativas, onde o menor $\mathrm{pH}$ encontrado foi 5,53 (G3) e o maior 6,74(G4) uma diferença de mais de 21,8\%. Diante dos diferentes pH das medicações estudadas, o cirurgião-dentista ao prescrever medicações, deve se atentar aos $\mathrm{pH}$ que aproximam dos níveis críticos de desmineralização dentária principalmente em pacientes com risco moderado e alto de cárie.

Palavras-chave: Hidrogênio; Erosão dentária; Carie dentária.

\begin{abstract}
The success of dental treatments, especially in pediatric dentistry, is directly linked to the treatment plan, where we must consider the patient as a whole, inserted within a family context and influenced by their social reality. Within these aspects, minimally invasive treatments and especially pain control are essential for establishing a harmonious relationship between patient, professional and family. Dentists can use an extensive list of medications that can help to control pain, among the most used analgesics, we highlight Dipyrone. For this study, 04 samples of the analgesic Dipyrone were used, which were divided according to the manufacturers: G1 - Medley (generic), G2 - Neo Química, G3 - EMS and G4 - Medley (Novalgina). After handling the medications according to the manufacturer, $\mathrm{pH}$ samples from each laboratory were measured at different times (immediately, 48 hours and 7 days). After analysis of variance and Tukey's test, the results showed that no statistically significant differences were found in the $\mathrm{pH}$ at different times in the same laboratory, but when considering the different laboratories, they all showed statistically significant
\end{abstract}


differences, where the lowest $\mathrm{pH}$ found was 5, 53 (G3) and the largest $6.74(\mathrm{G} 4)$ a difference of more than $21.8 \%$. Given the different $\mathrm{pH}$ of the studied medications, when prescribing medications, dentists should pay attention to $\mathrm{pHs}$ that approach critical levels of dental demineralization, especially in patients with moderate and high risk of caries.

Keywords: Hydrogen; Tooth erosion; Dental caries.

\section{Resumen}

El éxito de los tratamientos odontológicos, especialmente en odontopediatría, está directamente ligado al plan de tratamiento, donde debemos considerar al paciente como un todo, insertado en un contexto familiar e influenciado por su realidad social. Dentro de estos aspectos, los tratamientos mínimamente invasivos y especialmente el control del dolor son fundamentales para establecer una relación armoniosa entre paciente, profesional y familia. Los odontólogos pueden utilizar una extensa lista de medicamentos que pueden ayudar a controlar el dolor, entre los analgésicos más utilizados, destacamos la Dipirona. Para este estudio se utilizaron 04 muestras del analgésico Dipirona, que se dividieron según los fabricantes: G1 - Medley (genérico), G2 - Neo Química, G3 - EMS y G4 - Medley (Novalgina). Después de manipular los medicamentos de acuerdo con el fabricante, se midieron las muestras de $\mathrm{pH}$ de cada laboratorio en diferentes momentos (inmediatamente, 48 horas y 7 días). Luego del análisis de varianza y la prueba de Tukey, los resultados mostraron que no se encontraron diferencias estadísticamente significativas en el pH en diferentes momentos en un mismo laboratorio, pero al considerar los diferentes laboratorios, todos mostraron diferencias estadísticamente significativas, donde el pH más bajo encontrado fue 5, 53 (G3) y el mayor 6,74 (G4) una diferencia de más del 21,8\%. Dado el diferente $\mathrm{pH}$ de los medicamentos estudiados, a la hora de prescribir medicamentos, los odontólogos deben prestar atención a $\mathrm{pH}$ que se acerquen a niveles críticos de desmineralización dental, especialmente en pacientes con riesgo moderado y alto de caries.

Palabras clave: Hidrógeno; Erosión de los dientes; Caries dental.

\section{Introdução}

O cirurgião-dentista, em especial o Odontopediatra, tem um papel indispensável no cotidiano das crianças, haja vista seu conhecimento acerca das doenças que acometem a cavidade bucal e sua responsabilidade perante aos pais e responsáveis principalmente na primeira infância. O Diagnóstico precoce e o tratamento preventivos são fatores responsáveis pelo sucesso dos tratamentos em crianças (Jacob \& Iwasaki, 2014).

As medicações têm sido um ótimo coadjuvante na relação paciente e cirurgião dentista, prevenindo e tratando com sucesso casos de infecções, de dor e de modo profilático (Valença, Medeiros \& Sousa, 2009). A precisão da quantidade medicamentosa é crucial no tratamento, evitando assim, a sobrecarga do organismo e até mesmo a hipersensibilização. A ingestão de medicamentos sem acompanhamento e prescrição pode acarretar uma super resistência microbiana, na qual o composto não possui mais ação sobre aquela determinada cadeia, se tornando cada vez mais perigoso atualmente (Bispo et al, 2019).

A forma e frequência de administração das drogas tem relevância, pois nos períodos noturnos onde o corpo entra em um estado de inercia, a salivação é diminuída e consequentemente, a capacidade tampão da saliva. Ambos quando relacionados a má higiene bucal, geram riscos de aparecimentos de lesões cariosas e erosão dental (Freitas et al, 2016). Para Leite et al, (2011), a maioria dos medicamentos criados para o uso infantil, possuem alta concentração de açúcares, elevada acidez e um baixo potencial de Hidrogênio $(\mathrm{pH})$.

A cárie dental é possui etiologia multifatorial e se desenvolve a partir da desmineralização da superfície dental, causada por diversos fatores intrínsecos e extrínsecos. Sua ocorrência se da através de três fatores chaves: microorganismos cariogênicos, substrato fermentável (como a sacarose) e um hospedeiro vulnerável (Losso et al, 2009). O mais importante na realização de uma prescrição odontopediatrica é conhecer o paciente como um todo, bem como suas condições e morfologias. Tendo em vista que as crianças têm o metabolismo e as sequencias de desenvolvimentos diferente dos adultos (Bispo et al, 2019).

Dentre as medicações utilizadas na infância estão os antibióticos e analgésicos. Esses últimos não necessitam de receitas especiais para a compra e podendo ser adquiridos facilmente em qualquer farmácia, já que são indicados para o controle da dor. Dessa forma, a correta escolha medicamentosa, pode determinar maior êxito no tratamento dos pacientes (Carmo et al, 2009). 
A prescrição medicamentosa em odontopediatria deve ser realizada de forma minuciosa, compreendendo todas as fases de desenvolvimento de uma criança, bem como seus aspectos emocionais e físicos, peso, histórico médico e até mesmo familiar (Valença, Medeiros \& Sousa, 2009).

É de suma importância conhecer e analisar os diversos grupos de fármacos presente no mercado, devido ao seu potencial de ação e toxicidade no organismo. O padrão-ouro pré-estabelecidos nas bulas, muitas das vezes se tornam eficaz e confiável no tratamento. Contudo, não anula o fato de que cada paciente deve ser tratado de forma única e especial, sendo proposto um tratamento específico (Bispo et al, 2019).

A grande parcela dos fármacos contém açucares, fator este que é primordial no desencadeamento de lesões de cárie, quando aliados a má higiene bucal. Os açucares são adicionadas as substâncias medicamentosas com a finalidade de inibir o gosto desagradável das mesmas, favorecendo assim, a adesão das bactérias que por sua vez, degradam a superfície dental (Freitas, Moreira \& Massingnan 2016). De acordo com Silva et al, (2010) o uso dos açúcares, em especial a sacarose é bastante difundido por conta do seu baixo custo e fácil manipulação. Além disso, essa atua diretamente na superfície lisa dos dentes.

A sacarose é o edulcorante mais difuso e utilizado nas medicações de um modo geral, compondo de 11 a $62 \%$ do total de componentes (Marquezan et al, 2006). Balbani, Stelzer e Montovani (2006) relatam que a sacarose é um ótimo coadjuvante as medicações devido ao seu baixo custo, agindo como antioxidante e conservante e melhorando a viscosidade das soluções liquidas e tornando-as mais palatáveis.

Passos, Freitas e Sampaio (2008) apontam que a carie por medicamentos tem características de carie rampante, que afeta diretamente incisivos superiores e inferiores, e as superfícies oclusais dos molares que de acordo com Barreto e Gomes (2018) possuem superfícies irregulares, o que favorece aparecimento de biofilme e em consequência o aparecimento do Streptococcus Mutans, que é a bactéria pioneira no processo de colonização. Após aderir o esmalte dentário, se ocupar e formar uma barreira espeça, as bactérias liberam ácidos favorecendo a desmineralização do esmalte e dentina quando em condições favoráveis.

Crianças que fazem o uso prolongado de medicamentos odontopediatricos devido a problemas médicos crônicos, estão potencialmente mais propicias ao desenvolvimento da doença carie (Silva \& Santos,1994).

Para Nogueira et al, (2015), a automedicação casou diversos transtornos relacionados à saúde da populacional, sendo o mais grave deles, a resistência microbiana, além desta, outras patologias relacionadas com o uso indiscriminado de medicamentos podem surgir, tais como diminuição de fluxo salivar e perda de mineral dentário.

Outro processo de destruição do esmalte dentário é a erosão dentária que de acordo com Oliveira et al, (2019) é caracterizada como perda progressiva, irreversível dos tecidos dentários e que acontece sem envolvimento bacteriano (Maltarollo et al, 2020).

Para Gaetti-Jardim et el, (2013) a saúde bucal é um estado completo de harmonia e higidez ligada à saúde do paciente, desta forma, a instrução de higiene oral e a prevenção tornam-se fatores chave no sucesso do tratamento (Freitas et al,2016). Segundo Gomes e Silva (2010) estimular o paciente a praticar o autocuidado é primordial no controle da placa, utilizando-se de métodos simples e eficazes como a escovação e o uso do fio dental.

Toda e qualquer criança a ser medicada requer atenção, diferentemente das prescrições de destinadas ao adulto. Sendo essencial conhecer e calcular as dosagens e formulações disponíveis, para alcançar a excelência do tratamento. O cirurgião dentista detém a responsabilidade de uma satisfatória saúde bucal do paciente diante de uma correto diagnostico e prescrição (Valença, Medeiros \& Sousa, 2009).

Atualmente utilizam-se de técnicas menos invasivas, garantindo mais segurança as crianças e diminuindo significamente o nível de estresse e dor no consultório. Técnicas essas, que envolve instrumentos manuais e materiais de fácil manuseio (Villar et al, 2020). Com isso, as técnicas minimamente invasivas ganham notoriedade na atualidade devido a sua 
conduta clínica. A qual, se dá pela máxima preservação do órgão dental, não causando injúrias, alterando os modelos dogmáticos da odontologia antiga, gerando promoção e manutenção da saúde oral. As técnicas são acompanhamentos de lesões cariosas não cavitadas, manutenções de manchas brancas, confecções de preparos restauradores e controle da doença (Tumenas et al, 2014).

Para Passos, Freitas e Sampaio (2008) a higiene bucal adequada deve ser realizada logo após a ingestão de medicações odontopediatricas, sendo o passo mais importante na diminuição da propagação da doença cárie. As ações de higiene oral bem como o acompanhamento do cirurgião dentista devem ser seguidas à risca, promovendo assim, o bem estar do paciente.

Araújo , Lião e Alcantara (2017) relatma que a falta de conhecimento das medicações odontopediatricas sobre seu potencial cariogênico demanda uma necessidade ainda maior de agências reguladoras, tais como a vigilância sanitária com a finalidade de implantar políticas públicas e metodologias de análises para os devidos esclarecimentos.

O conhecimento acerca das medicações liquidas prescritas para crianças, seus componentes e o potencial de dano as estruturas dentárias, podem ser o fator primordial na prevenção da carie e da erosão dental (Freitas, Moreira \& Massignan, 2016).

O presente trabalho tem por objetivo avaliar o potencial de hidrogênio dos analgésicos (Dipirona) solução para uso pediátrico.

\section{Metodologia}

Esta pesquisa foi realizada no laboratório de Bioquímica da Centro Universitário Aparício Carvalho (FIMCA, Rondônia), e por apresentar apenas etapas laboratoriais e sem envolvimento de seres humanos foi dispensado da submissão ao Comitê de Ética e Pesquisa. Trata-se de uma pesquisa quantitativa, Segundo Estrela (2018) neste tipo de pesquisa faz-se a coleta de dados numéricos por meio do uso de medições de grandezas. Os Analgésicos s foram divididos em 04 grupos de acordo com o Laboratório de Fabricação conforme tabela 1.

Tabela 1. Amostras e Grupos.

\begin{tabular}{|c|c|c|c|c|}
\hline Grupos & Laboratório & Nome comercial & $\begin{array}{c}\text { Lote / } \\
\text { Fabricação }\end{array}$ & Composição*** \\
\hline G1 & Medley & $\begin{array}{l}\text { Dipirona } \\
\text { (genérico) }\end{array}$ & $\begin{array}{l}\text { ARA01542 04/20 } \\
\text { ARA02088 05/20 }\end{array}$ & $\begin{array}{l}\text { Aroma de acerola, caramelo, edeato de sódio, fosfato } \\
\text { de sódio dibasico dodecaidratado, fosfato de sódio } \\
\text { monobásico monoidratado, glicerol, metabissulfito de } \\
\text { sódio, metilparabeno, propilparabeno, sacarina sódica } \\
\text { di-hidratada e água purificada. }\end{array}$ \\
\hline G2 & Neo Química & $\begin{array}{l}\text { Dipirona } \\
\text { (genérico) }\end{array}$ & $\begin{array}{l}\text { B20E2581 05/20 } \\
\text { B20G0746 09/20 }\end{array}$ & $\begin{array}{l}\text { Edetato dissodico, sorbitol, metabissulfito de sódio e } \\
\text { água. }\end{array}$ \\
\hline G3 & EMS & $\begin{array}{l}\text { Dipirona } \\
\text { (genérico) }\end{array}$ & $\begin{array}{l}\text { L2A5314 01/21 } \\
\text { L1U4708 07/20 } \\
\text { L2A5314 01/21 }\end{array}$ & Ácido cítrico, sorbitol e água purificada \\
\hline G4 & Medley & Novalgina ${ }^{\circledR}$ & $\begin{array}{l}\text { 9RA05340 01/21 } \\
\text { ARA01542 02/21 } \\
\text { ARA02088 02/21 }\end{array}$ & $\begin{array}{l}\text { Aroma de acerola, caramelo, edetato de sódio, fosfato } \\
\text { de sódio dibasico dodecaidratado, fosfato de sódio } \\
\text { monobásico monoidratado, glicerol, metabissulfito de } \\
\text { sódio, metilparabeno, propilparabeno, sacarina sódica } \\
\text { di-hidratada e água purificada. }\end{array}$ \\
\hline
\end{tabular}

** Composição de acordo com a bula fornecida juntamente com cada medicamento. Fonte: Autores. 
Com o intuito de se aferir o pH das medicações supracitadas, foi utilizado o Aparelho $\mathrm{PH} 20$ value $\mathrm{pH}$ tester da marca CHEESELAB, Lote T211117058 (Figura 1).

$\mathrm{O}$ pH de cada medicamento foi determinado à temperatura controlada de $24^{\circ} \mathrm{C}$, usando um medidor de $\mathrm{pH}$ eletrônico. O equipamento com uma precisão de 0,1 foi calibrado de acordo com as instruções do fabricante utilizando soluções padrões de tampão de $\mathrm{pH} 7$ e pH 4.

Figura 1. Aparelho phmetro.

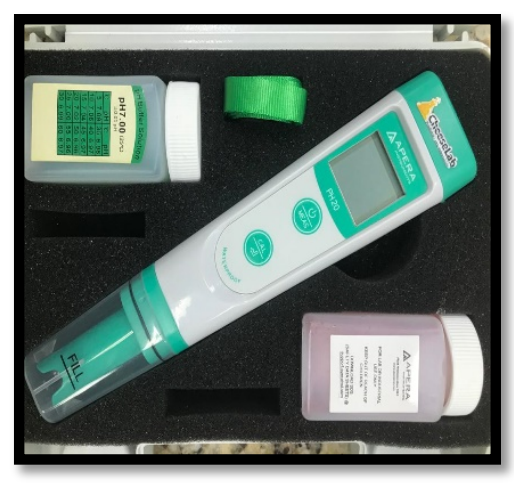

Fonte Autores.

As realizações das medidas foram realizadas em 03 momentos diferentes com intuito de verificar se existiam variação do $\mathrm{pH}$ com o passar do tempo (Tabela 2), desta forma, os frascos dos analgésicos eram abertos de acordo com os Grupos e colocado 20ml da solução em Becker de vidro (Figura 2). O pHmêtro era ligado e colocado em contato com o medicamento por 2 minutos, para que o aparelho fizesse uma leitura precisa. Eram realizadas 10 medidas de cada grupo para a obtenção dos valores de $\mathrm{pH}$, onde os mesmos eram anotados em planilha de Excel para posterior análise estatística (Figura3).

Tabela 2. Quantidades de amostras coletadas em diferentes momentos.

\begin{tabular}{cccc} 
Grupos & T0 - Imediato & T1- 48 horas & T2 -7 dias \\
\hline G1 & $(n=10)$ & $(n=10)$ & $(n=10)$ \\
\hline G2 & $(n=10)$ & $(n=10)$ & $(n=10)$ \\
\hline$G 3$ & $(n=10)$ & $(n=10)$ & $(n=10)$ \\
\hline G4 & $(n=10)$ & $(n=10)$ & $(n=10)$
\end{tabular}

Fonte Autores. 
Figura 2. Medicação em Becker de vidro.

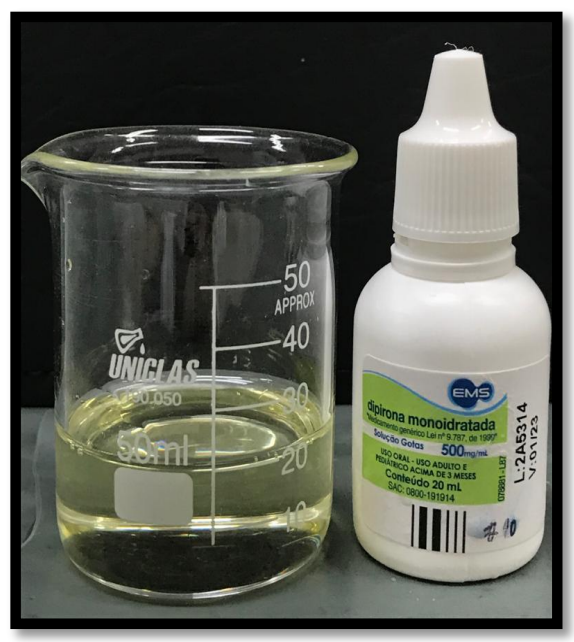

Fonte: Autores.
Figura 3. Aferição de pH.

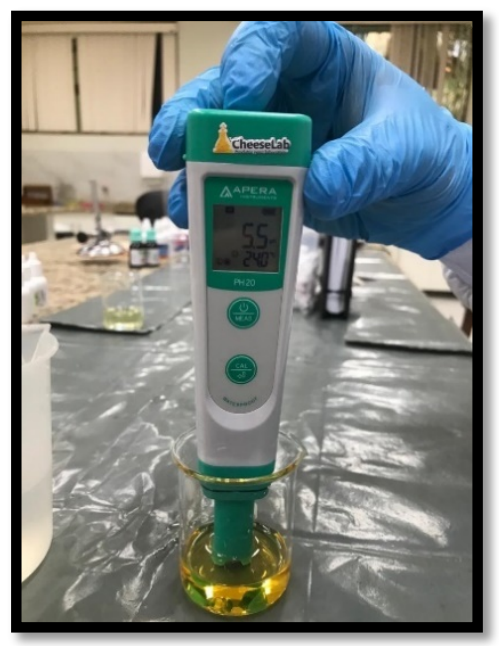

Fonte: Autores.

Após a realização de todas as aferições, os dados foram submetidos a análise de variância e Teste complementar de Tukey.

\section{Resultados}

Após a análise estatística (Anova e Teste complementar de Tukey) foram observados os seguintes resultados:

Tabela 3. Análise Descritiva das amostras e $\mathrm{pH}$ em seus respectivos tempos.

\begin{tabular}{clccc}
\hline & Laboratório & pH Imediato & pH 48 horas & pH 7 dias \\
\hline \multirow{2}{*}{ Média } & Medley & 6.03 & 6.03 & 6.03 \\
& Neo Química & 5.58 & 5.53 & 5.54 \\
& EMS & 5.90 & 5.82 & 5.81 \\
& Medley “Novalgina” & 6.73 & 6.74 & 6.72 \\
\hline \multirow{2}{*}{ Desvio Padrão } & Medley & 0.0949 & 0.0949 & 0.0949 \\
& Neo Química & 0.114 & 0.0949 & 0.0843 \\
& EMS & 0.105 & 0.103 & 0.110 \\
& Medley “Novalgina” & 0.0949 & 0.0966 & 0.0919 \\
\hline
\end{tabular}

Fonte: Autores.

Tabela 4. Variação de $\mathrm{pH}$ de um mesmo medicamento em diferentes momentos.

\begin{tabular}{llllll}
\hline & Soma dos quadrados & df & Média dos quadrados & F & p \\
\hline Diferentes momentos & 0.0287 & 2 & 0.01433 & 1.543 & 0.221 \\
Dif. Momentos * Laboratório & 0.0360 & 6 & 0.00600 & 0.646 & 0.693 \\
Residual & 0.6687 & 72 & 0.00929 & & \\
& & & & & \\
\hline
\end{tabular}

(Sem diferença estatística pois $\mathrm{p}>0,05)$. Fonte: Autores. 
Tabela 5. Variação considerando apenas laboratórios.

\begin{tabular}{llllll}
\hline & Soma dos Quadrados & df & Média dos quadrados & F & p \\
\hline Laboratório & 22.649 & 3 & 7.5497 & 713 & $<.001$ \\
Residual & 0.381 & 36 & 0.0106 & & \\
\hline
\end{tabular}

(Diferença estatística pois p é $<0,05$ ). Fonte: Autores.

Tabela 6. Teste de Tukey - Comparação entre Laboratórios.

\begin{tabular}{|c|c|c|c|c|c|c|c|}
\hline \multicolumn{3}{|l|}{ Comparação } & \multirow[b]{2}{*}{ Diferença } & \multirow[b]{2}{*}{ SE } & \multirow[b]{2}{*}{ df } & \multirow[b]{2}{*}{$\mathbf{t}$} & \multirow[b]{2}{*}{$\mathbf{p}_{\text {tukey }}$} \\
\hline Laboratório & & Laboratório & & & & & \\
\hline \multirow[t]{3}{*}{ Medley } & - & Neo Química & 0.480 & 0.0266 & 36.0 & 18.06 & $<.001$ \\
\hline & - & EMS & 0.187 & 0.0266 & 36.0 & 7.02 & $<.001$ \\
\hline & - & Medley & -0.700 & 0.0266 & 36.0 & -26.34 & $<.001$ \\
\hline \multirow[t]{2}{*}{ Neo Química } & - & EMS & -0.293 & 0.0266 & 36.0 & -11.04 & $<.001$ \\
\hline & - & Medley & -1.180 & 0.0266 & 36.0 & -44.40 & $<.001$ \\
\hline SEM & - & Medley & -0.887 & 0.0266 & 36.0 & -33.37 & $<.001$ \\
\hline
\end{tabular}

(Diferença estatística pois p é $<0,05$ ). Fonte: Autores.

Gráfico 1. Curva de variação entre as medicações, conforme os tempos observados.

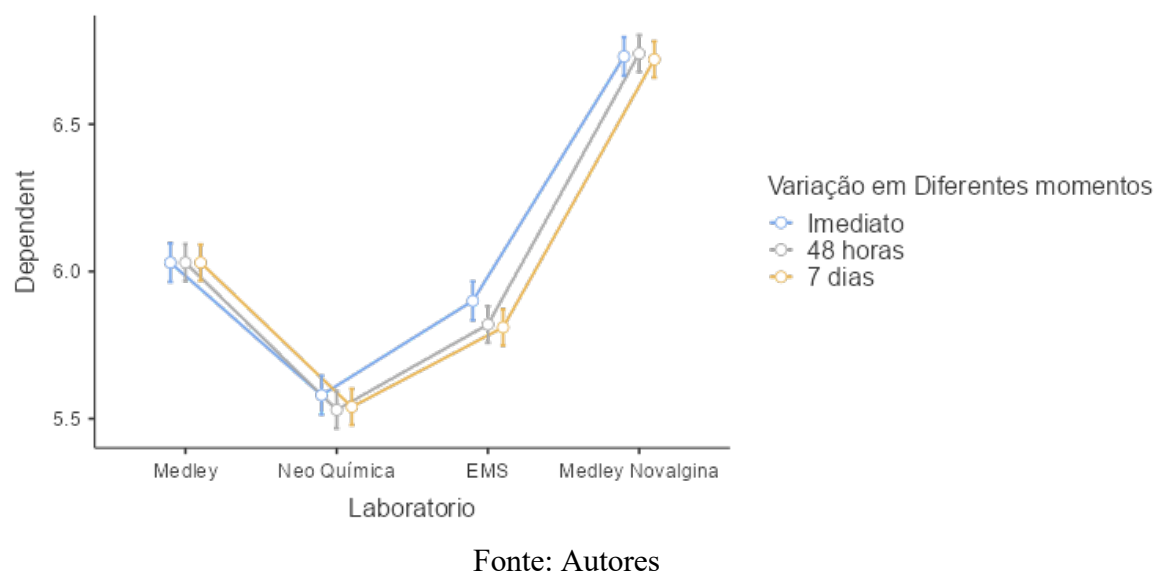

\section{Discussão}

Os medicamentos pediátricos, em sua grande parcela, são coloridos, adoçados e aromatizados por meio de um conjunto de adjuvantes, os excipientes, que tem por finalidade "camuflar" o gosto desagradável das medicações, tornando-as mais palpáveis para as crianças. Os xaropes por sua vez, são concentrados de soluções aquosas contendo açúcares que servem de veículos de sabor agradável para substâncias ativas (Freire, Campos \& Martins, 2018).

Oliveira et. al, (2019) afirmam em seu estudo que medicamentos infantis causam nos dentes decíduos, principalmente, alterações na superfície do esmalte, formação de crateras, perda de cálcio e diminuição da dureza. Além do pH baixo, essas medicações apresentam viscosidade e elevada acidez titulável, tornando-se a causa principal da desmineralização dentaria. Vale ressaltar que os dentes decíduos são conhecidos por serem menos mineralizados que os dentes permanentes e, particularmente 
como a superfície do esmalte dos dentes decíduos não é tão madura quanto a dos dentes permanentes, é mais sujeita a cáries dentárias (Johansson et al, 2001).

Freire, Campos e Martins (2018) indicam que o pH das medicações líquidas está relacionado com a estabilidade das substâncias presentes, podendo ocorrer decomposição química em razão do pH inadequado, comprometendo assim, a sua ação farmacológica.

Outra consideração que nos chama atenção é que o uso das dipironas, muitas vezes é prescrita com seu uso de 6 em 6 horas ou até mesmo de 04 em 04 horas, o que inevitavelmente irá ter uma dose durante a noite ou madrugada. Dessa forma, o fluxo salivar diminuído pode aumentar a chances de desmineralização associado a falta de higiene bucal. De acordo com Sunitha et al, (2009) as medicações líquidas pediátricas causam queda no pH da placa que é suficiente para causar descalcificação dentro de 2 a 10 minutos após sua exposição incial aos dentes.

No presente trabalho verificamos que com o passar do tempo, ou seja, depois de aberto o frasco da medicação (Dipirona) o efeito "tempo" não afeta o $\mathrm{pH}$ nos momentos aferidos, por isso, para o mesmo laboratório, não foram encontradas diferenças estatisticamente significativas de $\mathrm{pH}$, seja imediatamente, com 48 horas ou 7 dias, o que demonstra dentro deste período uma estabilidade.

Quando comparamos os diferentes laboratórios, que vendem a mesma medicação, Dipirona, encontramos diferenças consideráveis entre cada laboratório. O que pode ser explicado pelas diferentes composições informadas na bula de cada medicação (Tabela 1) além disso, vale ressaltar que os Grupos: G2 e G3 que apresentaram menor pH o medicamento era armazenado em frascos de plástico, enquanto os Grupos G1 e G4 em frascos de vidro.

Ao analisarmos o Grupo com menor pH, G2, percebemos que a bula da medicação é a que trás menos informações sobre a composição do medicamento, seguida pelo medicamento do Grupo G3.

O pH observado no grupo G2 atingiu, os menores níveis, variando de 5,58 quando avaliado imediatamente, 5,53 nas 48 horas e 5,54 com 7 dias. Apesar de não existir diferença entre os 3 momentos, de acordo com Guerra e Oliveira (2013) o nível crítico do pH para dissolução do esmalte dental é de 5,5, ou seja, quando esse nível crítico é atingindo a cavidade bucal se torna ambiente propicio para o aparecimento da cárie.

O grupo G2 que apresentou os menores níveis de ph possui o sorbitol em suas formulações, juntamente com edetato dissódico que atua como conservante e oxidante, atuando melhor em ph que varia de 5 a 7 (Azulay \& Azulay 2011).

O Grupo G3 que também apresentou pH menores que 6, possui o sorbitol combinado com o ácido cítrico que também atua como conservante. De acordo com Fuller e Johnson (1977) a atividade erosiva do ácido cítrico foi comprovada.

Em relação aos maiores níveis de pH: G1 e G4, o que nos chama a atenção são que os dois são produzidos pelo mesmo laboratório, sendo G1 o genérico e o G4 conhecido pelo seu nome comercial (Novalgina).

O Nível mais elevado de pH foi observado em G4 (Novalgina) 6,74 enquanto o G1 do mesmo laboratório, conhecido como genérico, apresentou pH de 6,03.

Uma das hipóteses para os resultados observados poderia ser explicada pelo armazenamento em frasco de vidro, o que confere maior estabilidade e proteção contra a luz. Outra, seria sua formulação mais complexa, que utilizar diversas substâncias além de ter como açúcar a sacarina (Tabela 1).

Independente dos $\mathrm{pH}$ observados, variando de 5,5 e até 6,74 em nosso estudo, segundo Mahmoud e Omar (2018), os resultados de sua pesquisa revelou que analgésicos e antibióticos com pH entre 5,68 e 6,22 foram capazes de causar irregularidades, deixar as superfícies rugosas com numerosos poros de diferentes tamanhos e profundidades quando analisado por Microscopia Eletrônica de Varredura. Desta forma fica evidente o potencial erosivo e cariogênico da Dipirona, o que corrobora com os estudos de Tupalli et al, 2014. 
As bulas dos medicamentos analisados, não especificaram nenhuma informação valiosa sobre o seu pH ou quantidade de açúcar, corroborando com os achados de Girish Babu et. al,(2014).

Independente do Grupo analisado, nenhuma bula informava a quantidade de açúcar, nem possíveis riscos a cárie e erosão dentária. Estas informações deveriam estar presentes já que estes medicamentos são vendidos sem qualquer controle.

\section{Conclusão}

O presente estudo conclui que existem diferenças estatisticamente significativas do $\mathrm{pH}$ entre Dipironas de diferentes laboratórios. Valores próximos ao considerável crítico para o início do processo de desmineralização foram observados no Grupo G2. Os medicamentos que utilizavam o sorbitol em sua formulação possuíam os níveis mais baixos de pH.

O Cirurgião Dentista deve conhecer as características das medicações que prescreve para otimizar o sucesso do tratamento odontológico, além disso, mais estudos relacionando $\mathrm{pH}$, medicações, cárie dental e erosão devem ser realizados para elucidar possíveis duvidas durante a prescrição de medicação na Odontologia.

\section{Referências}

Araújo, N. V. P, Lião, L. M. \& Alcantara, G. B. (2017) Quantificação de sacarose em formulações farmacêuticas líquidas de uso oral pela técnica de RMN de 1H. Docplay, Goiás, 6,1-15.

Azulay, R. D.; \& Azulay, L. (2011) Dermatologia. 5. ed. São Paulo: Guanabara-Koogan,

Balbani, A. P. S.; Stelzer, L. B.; \& Montovani, J. C. (2006) Excipientes de medicamentos e as informações da bula. Revista Brasileira de Otorrinolaringologia, 72(3),400-406

Bispo, A. R., Melo, A. L. B, Lamenha, R., Pugliesi, D. M. C, \& Santos Júnior, V. E. (2019) Análise da confiabilidade de fórmulas para o cálculo de dose de antibacterianos em odontopediatria. Revista da Faculdade de Odontologia-UPF, 24(1), 14-21.

Barreto, L. S. D. C. \& Gomes, M. P. (2018). Conhecimento da doença cárie e a relação streptococcus mutans na microbiota salivar (pcr): uma revisão de literatura. Revista Fluminense de Odontologia,48(2), 1-12.

Carmo, E. D, Amadei, S. U., Pereira, A. C., Silveira, V. A. S, Rosa, L. E. B, \& Rocha. R. F. (2009) Prescrição medicamentosa em odontopediatria. Revista de Odontologia da UNESP, 38(4) 256-62.

Costa, C. C., Almeida, I. C. S., Raymundo, M. S., \& Felt, R. (2004) Análise do pH endógeno, da acidez e da concentração de sacarose de medicamentos pediátricos. Rev. odonto ciênc, 164-169.

Estrela, C. (2018). Metodologia Científica: Ciência, Ensino, Pesquisa. Artes Médicas.

Freire, T. F. C.; Campos, E. D. J.; \& Martins, G.B. (2018) Avaliação in vitro do potencial cariogênico e erosivo de corticosteroide de uso tópico. Revista de Ciências Médicas e Biológicas, 17(3), 386-391.

Freitas, M. P.R, Moreira, J. M., Massignan, C., Cardoso, M., \& Bolan, Michele. (2016) Conhecimento dos pediatras sobre os efeitos dos medicamentos na cavidade bucal. Arquivos Catarinenses de Medicina, 45(4) 67-77.

Fuller, J. L., \& Johnson, W. W. (1977). Citric acid consumption and the human dentition. J Am Dent Assoc.,95-80.

Gaetti-Jardim, E., Setti, J. S., Cheade, M. F. M., \& Mendonça, J. C. G. (2013). Atenção odontológica a pacientes hospitalizados: revisão da literatura e proposta de protocolo de higiene oral. Revista de Atenção à Saúde, 11(35), 31-36.

Girish Babu, K. L.; Doddamani, G. M, Kumaraswamy Naik, L. R; \& Jagadeesh, K. N. (2014). Pediatric liquid medicaments - Are they cariogenic? An in vitro study. J Int Soc Prevent Communit Dent ,4:108-12

Gomes, V. E., \& Silva, D. D. (2010) A importância do controle de placa dental na clínica odontológica. Arquivos em Odontologia, $46(1), 22-27$.

Guerra, C. F.; \& Oliveira, F.S (2013) Alterações bucais causadas pela bulimia nervosa. Monografia (Graduação em Odontologia) - Faculdade de Odontologia, FAPI, São Paulo.

Jacob, S. \& Iwasaki, K. K. (2014) A Influência Do Antibiótico Na Cárie Infantil: The Influence of Antibiotics in Child Caries. Brazilian Journal of Surgery and Clinical Research, 8(20) 68-74.

Johansson, A. K, Sorvari, R., Birkhed, D., \& Meurman, J. H. (2001) Dental erosion in deciduous teeth--an in vivo and in vitro study. J Dent 29:333-340.

Korb, A., Brambilla, D. K., Teixeira, D. C., \& Rodrigues, R. M (2011) Riscos para a saúde humana do uso de antibióticos na cadeia produtiva leiteira. Revista de Saúde Pública de Santa Catarina, 4(1) 21-36. 
Research, Society and Development, v. 10, n. 7, e43110716925, 2021

(CC BY 4.0) | ISSN 2525-3409 | DOI: http://dx.doi.org/10.33448/rsd-v10i7.16925

Leite F.; Leite, C.; Correia, A.; \& Pinto, M.;(2011) Medicamentos pediátricos e cáries dentárias - Percepções e atitudes de um grupo de tutores pediátricos em Vila Nova de Gaia. Revista Portuguesa de Estomatologia, Medicina Dentária e Cirugia Maxilofacial, Portugal, 52(4),193-199.

Losso, E. M, Tavares, M. C, Silva, J. Y. \& Urban, C. A. (2009) Severe early childhood caries: an integral approach. J pediatr, 85(4),295-300,.

Maltarollo, T. H.; Pedron, I. G.; Medeiros, J. M. F.; Kubo, H.; Martins, J. L.; \& Shitsuka, C. (2021) A erosão dentária é um problema! Research, Society and Development, [S. 1.], v. 9, n. 3, p. e168932723, 2020. DOI: 10.33448/rsd-v9i3.2723.

Marquezan, M., Marquezan, M., Pozzobon, R, \& Oliveira, M. (2006) et al. Avaliação do potencial cariogênico de medicamentos pediátricos.Saúde (Santa Maria), 32(1). 4-9.

Mahmoud,E .F.; \& Omar, O. M. (2018) Erosive and cariogenic potential of various pediatric liquid medicaments on primary tooth enamel: A SEM study. Dent Med Probl. 55(3),247-254.

Monteiro, D. R. S. (2016). Medicamentos pediátricos e cárie dentária: perceções e atitudes dos médicos de medicina geral e familiar. 2016 . Tese de Doutorado Universidade Católica Portoguesa. Lisboa. Portugal

Nogueira, J. S. E., Bonini, G. A. V. C., Mascaro, M. S. B., Imparato, J. C. P., \& Politano, G. T. (2015) Automedicação em crianças atendidas em centro de especialidades odontológicas na Amazônia. Revista da Associacao Paulista de Cirurgioes Dentistas, 69(4),369-375.

Oliveira, A. M., Lopes, A. G., Magalhães, T. C., Carlo, H. L., \& Carvalho, F. G. (2019) Os medicamentos infantis podem causar erosão na superfície de dentes decíduos? Revisão sistemática da literatura. HU Revista, 45(1), 65-75.

Passos, I. A, Freitas, C. H. S., \& Sampaio, F. C. (2008) Potencial cariogênico de medicamentos pediátricos-papel na etiologia da cárie dentária Cariogenic potential of pediatric medicines-role in the etiology of dental caries. Rev Inst Ciênc Saúde, 26(1), 125-129.

Silva, S. M. B; \& Santos, C. F. (1994). Medicamentos pediátricos e risco de cárie: uma revisäo. Rev. Fac. Odontol. Bauru, 15-21.

Silva, C. O., Reis, M. E. S., Satana, K. F. P., Maestri, F. P., Saakamp, C. J., \& Maestri, R. P. (2015). Avaliação Do Potencial Cariogênico De Anti-Histamínicos De Uso Pediátrico.Revista Eletrônica de Farmácia, 12(3)15-22.

Sunitha S, Prashanth G. M, Shanmukhappa, Chandu G. N, \& Subba Reddy, V. V. (2009). An analysis of concentration of sucrose, endogenous pH, and alteration in the plaque $\mathrm{pH}$ on consumption of commonly used liquid pediatric medicines. J Indian Soc Pedod Prev Dent.27:44-48.

Tupalli A. R, Satish, B., Shetty, B. R, Battu, S., Kumar, J. P, \& Nagaraju, B. (2014) Evaluation of the erosive potential of various pediatric liquid medica- ments: An in vitro study. J Int Oral Health. 6(1),59-65.

Tumenas, I., Pascottos, R., Saade, J. L., \& Bassani, M. (2014). Odontologia minimamente invasiva. Revista da Associacao Paulista de Cirurgioes Dentistas, 68(4), 283-295.

Valença, A. M. G; Medeiros, A. L; \& Sousa, S. A. (2009). Terapêutica medicamentosa adotada por cirurgiões-dentistas para pacientes pediátricos na atenção básica à saúde na cidade de João Pessoa-PB. Rev. bras. ciênc. saúde, 53-65.

Villar, B.B., Rodriguez, C. V., Canizares, V., \&Moreta, L. T. (2020) Técnicas en el manejo de la conducta del paciente odontopediátrico. Cient. dent. 17(1),2734 